\title{
Nephrectomy in a Patient with Medullary Sponge Kidney Associated with Severe Nephrocalcinosis and Nephrolithiasis: A Rare Condition
}

\author{
Landry Oriole Mbouché1,2, Achille Aurèle Mbassi2,3, Junior Mekeme Mekemee1,2, \\ Axel Stéphane Nwaha Makon', Pierre Joseph Fouda',2, Fru Fobuzshi Angwafo III1 \\ ${ }^{1}$ Department of Surgery and Specialities, Faculty of Medicine and Biomedical Sciences, Yaoundé, Cameroon \\ ${ }^{2}$ Department of Uro-Andrology, Yaoundé Central Hospital, Yaoundé, Cameroon \\ ${ }^{3}$ Department of Surgery, Higher Institute of Medical Technology, Yaoundé, Cameroon \\ Email: landrymbouche@gmail.com,drmbassi@yahoo.com, juniormekeme@yahoo.fr, nwahamakon@gmail.com, \\ foudapierrejoseph@gmail.com, asanji55@hotmail.com
}

How to cite this paper: Mbouché, L.O., Mbassi, A.A., Mekeme, M.J, Makon, A.S.N., Fouda, P.J. and Angwafo III, F.F. (2021) Nephrectomy in a Patient with Medullary Sponge Kidney Associated with Severe Nephrocalcinosis and Nephrolithiasis: A Rare Condition. Open Journal of Urology, 11, 332-341.

https://doi.org/10.4236/oju.2021.119032

Received: August 3, 2021

Accepted: September 13, 2021

Published: September 16, 2021

Copyright $\odot 2021$ by author(s) and Scientific Research Publishing Inc. This work is licensed under the Creative Commons Attribution International License (CC BY 4.0).

http://creativecommons.org/licenses/by/4.0/

\begin{abstract}
Background: Medullary sponge kidney (MSK) is a disturbance of renal development characterized by cystic dilation and diffuse precalyceal duct ectasia. The disease affects both genders in equal proportions and is generally diagnosed in adulthood, as a result of recurrent calcium nephrolithiasis and nephrocalcinosis. The most frequently encountered manifestations being renal colic, microscopic or macroscopic hematuria, and fever. The intravenous pyelogram is standard for diagnosis and metabolic workup is required to identify the underlying cause. The main goal of treatment is to prevent recurrence and disease progression. Though considered a benign condition, a nephrectomy may often be required in patients presenting late with irreversible complications and end-stage renal disease. Aim: To highlight and discuss the presentation and management of a rare case of nephrocalcinosis and nephrolithiasis secondary to the medullary sponge kidney. Case presentation: We report herein the case of a 56-year-old male with long-standing hematuria in whom a diagnosis of medullary sponge kidney disease was made and he underwent a left total nephrectomy. The postoperative course was uneventful. Conclusion: Nephrocalcinosis and nephrolithiasis are complications of MSK and can result in irreversible renal damage. A high index of suspicion is necessary for patients presenting with renal colic, recurrent urinary tract infections, or hematuria for prompt diagnosis and management.
\end{abstract}




\section{Keywords}

Medullary Sponge Kidney, Nephrolithiasis, Nephrocalcinosis, Nephrectomy

\section{Introduction}

Medullary sponge kidney (MSK) or Cacchi-Ricci disease represents a disturbance in renal development characterized by cystic dilation and diffuse precalyceal duct ectasia. These dilations are between 1 and $7 \mathrm{~mm}$ in size, frequently containing small calculi, and the communication between the respective ducts and calyces may be stenotic [1].

The true prevalence of MSK in the general population is not known, but is estimated to be less than $0.5 \%-1 \%$. MSK is much more frequent $(12 \%-20 \%)$ in recurrent renal calcium stone formers [2].

It has been claimed that MSK results from the coexistence of multiple tubular function defects in different nephron segments which points to the abnormal development (polarization) of the tubular epithelium, probably as a consequence of an abnormal "ureteric bud-metanephric mesenchyme", or receptor tyrosine kinase (RET)/glial cell line-derived neurotrophic factor (GDNF) interface [2].

MSK affects both genders and is generally diagnosed in young adults. The disease is typically observed in recurrent renal stone formers [2]. MSK can be asymptomatic but can present as nephrocalcinosis, urolithiasis, and recurrent urinary tract infections (UTI's) [3]. Uncommonly, in a few patients, the clinical course of MSK may be dominated by pain, which may, or may not, be associated with the spontaneous passage of stones or pyelonephritis [2]. Indeed, microhematuria and macrohematuria are naturally very frequent, generally associated with stone passage, though rarely, patients may experience frank, persistent hematuria apparently unrelated to the passage of stones [2].

We present and share our experience in the management of a complicated case of MSK in a 56-year-old patient with long-standing hematuria.

\section{Case Report}

A 56-year-old patient living in the northern region of Cameroon was referred for multiple lithiases of the left kidney. He complained of multiple episodes of total hematuria associated with left renal colic. There were no urinary symptoms or fever. His past history revealed repeated hematuria for the last 31 years. He has type 2 diabetes and has been followed for 14 years on oral antidiabetic drugs. There was no notion of urinary schistosomiasis or smoking. Clinical examination showed stable vital parameters with a blood pressure of $130 / 85 \mathrm{mmHg}$ and heart rate of 78 beats/minutes. Physical examination was unremarkable. In the absence of scintigraphy, we were unable to assess differential renal function although serum creatinine $(9.13 \mathrm{mg} / \mathrm{l})$ and urea $(0.33 \mathrm{~g} / \mathrm{l})$ were normal. A plain abdominal radiograph (Figure 1) showed multiple opacities on the left renal 
area. CT Urogram (Figure 2) showed left nephrocalcinosis and right renal lithiasis. It also showed accumulation of contrast medium in dilated ducts within the left renal medulla with the characteristic "bouquet of flowers" appearance. The ureter was not visible on the left side, but there was no obstruction on the right. Calcemia $(90.3 \mathrm{~g} / \mathrm{l})$ and natremia $(148.1 \mathrm{mEq} / \mathrm{l})$ were normal. In contrast, phosphoremia (51.87 IUI/l) and uricemia ( $72 \mathrm{mg} / \mathrm{l})$ were slightly elevated. Urine culture was sterile and urine electrolytes were not checked. We carried out a left total nephrectomy (Figure 3) via a flank approach under general anesthesia. The operative findings included 61 stones of various sizes, arranged in layers in the medulla and cortex as well as diffuse ectasia of the precalyceal ducts. This was strongly suggestive of Cacchi-Ricci disease (Figures 4-6). The intervention was hitch-free and the postoperative course was uneventful. Neither stone analysis

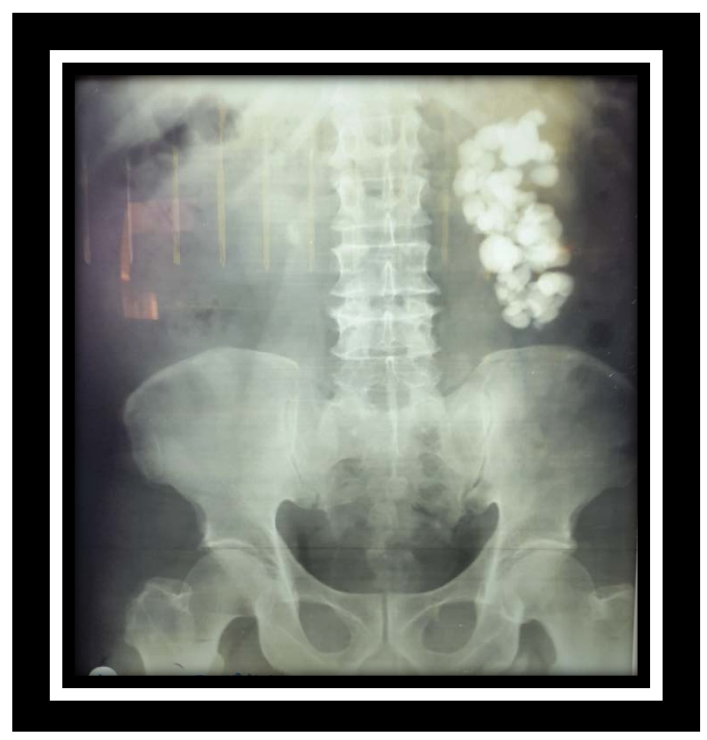

Figure 1. Plain abdominal radiograph showing multiple calcifications in left renal area.

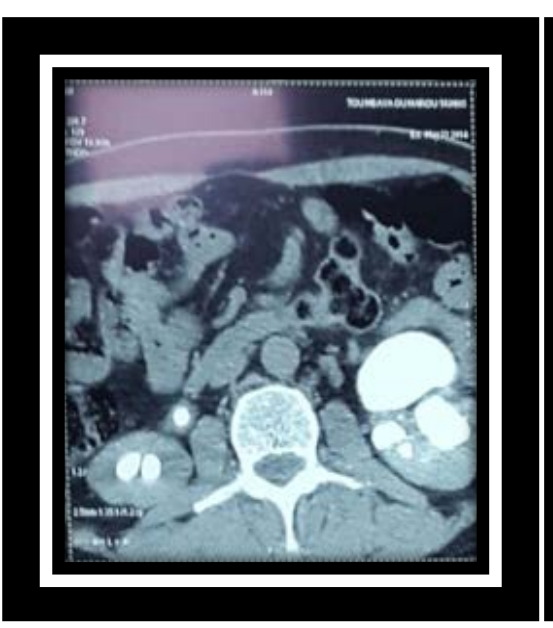

(A)

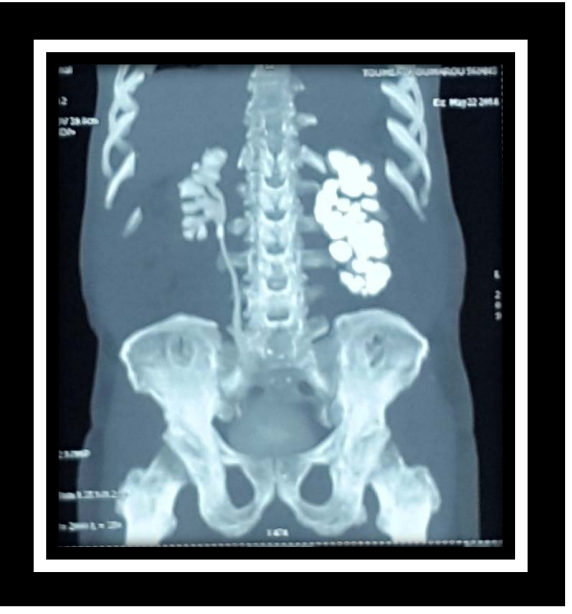

(B)

Figure 2. CT Urogram ((A) bilateral urolithiases; (B) "Bouquet of flowers" appearance during the excretory phase). 


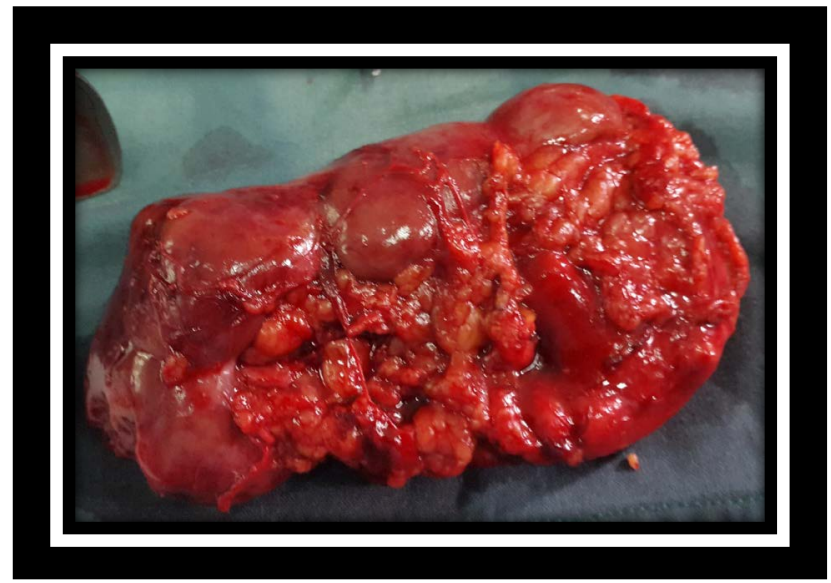

Figure 3. Left kidney specimen after nephrectomy.

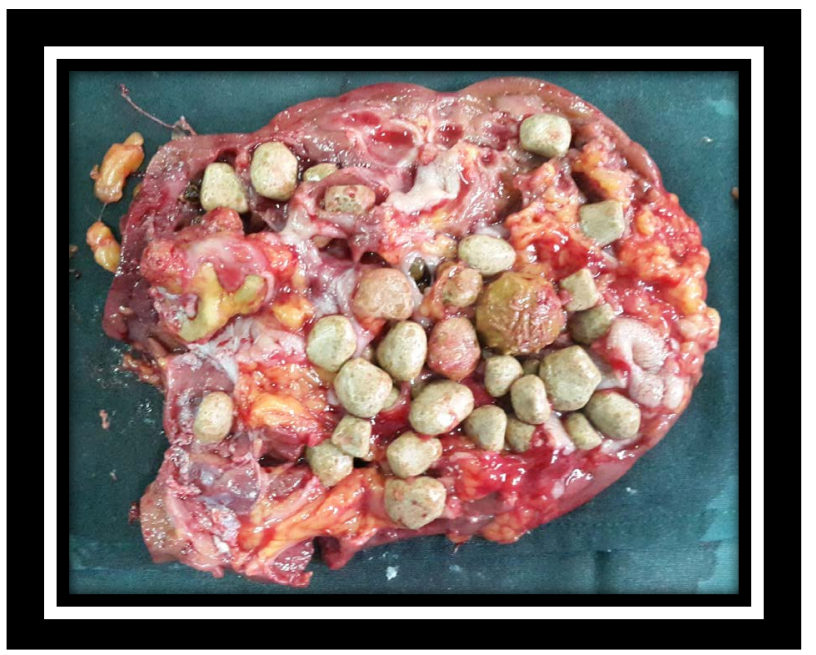

Figure 4. Nephrocalcinosis.

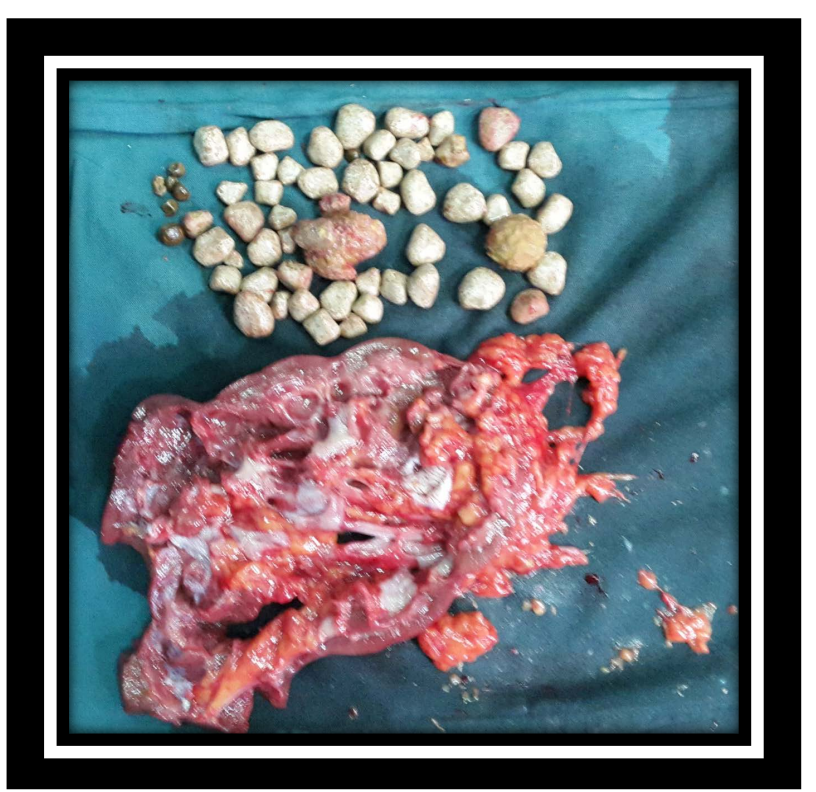

Figure 5. Precalyceal ectasia of left kidney. 


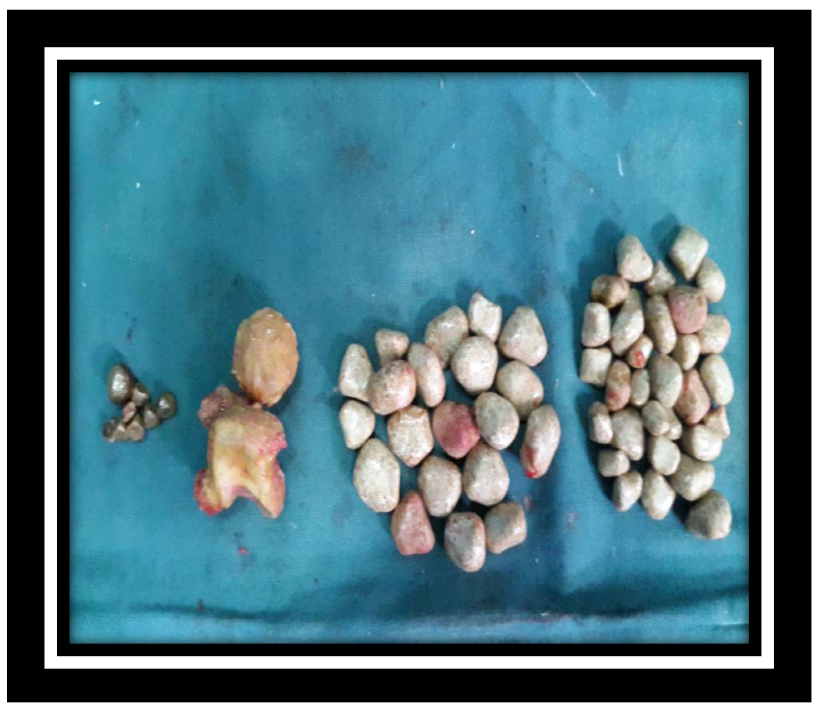

Figure 6. 61 stones from left kidney.

nor histopathology of the nephrectomy specimen was performed. The patient was discharged on postoperative day five. The patient was jointly reviewed with the nephrologist. Follow-up visits were done at 6 and 9 months with assessment of renal function and ultrasound imaging of the urinary tract during each visit. The right renal calculi were managed conservatively with high fluid intake and urine alkalinization using potassium citrate. Adverse prognosis was mitigated by strict follow up with regular control of renal function and screening for urinary tract infections as well as dietary modification.

\section{Discussion}

Theories behind the etiology of stone formation in MSK include urinary stasis in dilated tubules leading to increased crystal precipitation, renal leak, hypercalciuria resulting from impaired tubular function, and distal renal tubular acidosis and associated hypocitraturia resulting from impaired tubular function [4].

Any hypothesis on the pathogenesis of MSK should explain the concomitant occurrence of alterations in the ureteric bud derived pre-calyceal and collecting ducts (i.e. the "lower nephron"), such as "cysts", nephrocalcinosis and acidification and concentration defects, as well as in the metanephric blastema-derived nephron ("upper nephron"), such as proximal tubular defects [3].

As a disease itself, MSK can coexist with other renal developmental anomalies since it is considered a renal developmental malformation itself. Abnormalities in developmental genes including GDNF and RET as well as the association with Multiple Endocrine Neoplasia (MEN 2A) can lead to several different renal pathologies, extending beyond MSK [5].

Most patients with MSK are asymptomatic. MSK may have a sporadic prevalence of 5/10,000 to 5/100,000 for some authors while other sources state that the epidemiology of MSK is difficult to predict. This may be the result of missed diagnosis as MSK is known to have an indolent course and often asymptomatic 
presentation [5].

The diagnosis is highly suggested in light of investigations imposed by related complications such as renal stones, urinary tract infections, renal tubular acidosis or urine concentration defects. In some cases, MSK may be silent or take an indolent clinical course with an unremarkable history; very rarely though, the MSK patient may have a clinical course marked by colicky flank pain not necessarily associated with stone passage or macro haematuria [3].

The disease is clinically diagnosed in $0.2 \%$ to $20 \%$ of cases, the most frequently encountered manifestations being renal colic, micro- or macroscopic hematuria, and fever [1] [4]. Indeed, nephrolithiasis may also lead to frequent UTIs, pyelonephritis and hematuria and the differential diagnoses for MSK can be made more precisely when patients present with other renal manifestations, especially in groups at risk. These groups include patients between the age of 20 and 30, those with other renal malformations, high sodium diet, hyperparathyroidism, and family history of MSK [5]. It was recurrent hematuria and left renal colic that led our patient to consult. The diagnosis was however made late as the patient has had recurrent hematuria for the past 31 years.

Fabris et al. [3] reported that the disease affects both genders in equal proportions, is generally diagnosed in adulthood and results in recurrent calcium nephrolithiasis and nephrocalcinosis. Moreover, it was suspected that the diagnosis is made mainly in adulthood because of its progressive nature [3].

The vast majority of MSK patients develop medullary nephrocalcinosis which may be noticeable on ultrasound as renal pyramid hyperechogenicity of different intensity. Due to the small size of calcium salt deposits, they are usually not accompanied by acoustic shadows [6].

In patients with suspected MSK, further imaging techniques are required hence the ultrasound signs are not specific for this condition [7]. The gold standard for diagnosis of MSK remains IVU showing accumulation of contrast medium within papillary ducts.

However, other procedures such as CT, X-ray, and endoscopy can also be used [5]. Radiologic imaging makes it possible to confirm the diagnosis as well as identify complications as is the case when stones occupy the entire urinary tract. Indeed, it is a disease found in $12 \%$ to $20 \%$ of radiopaque renal stones composed predominantly of calcium oxalate. Nephrolithiasis can be observed in $70 \%$ of patients with MSK [5].

Typical radiographic images of MSK show collections of contrast media in dilated collecting tubules with the appearance of a papillary brush or bouquet of flowers [8] [9]. Contrast medium collection in papillary ducts leads to this classic image which when present displays either a pattern of bouquets, brush-like striation, or linear striations [8]. In our case, the first plain abdominal film done by the patient showed the characteristic "bouquet of flowers" appearance.

Our patient's CT Urogram showed nephrocalcinosis and nephrolithiasis with complete obstruction on the left, but no obstruction on the right. However, some 
authors stated that the replacement of IVU by CT imaging is a drawback in MSK management due to CT being unable to properly demonstrate the classic signs of MSK [8] [10] [11]. CT imaging has been proven to better establish the diagnosis of most urologic conditions, but this, unfortunately, does not apply to MSK. However, Multi-detector computed tomography can accurately diagnose MSK comparable to IVU [8].

As another tool for diagnosis, endoscopy has been shown to properly establish a diagnosis of MSK by observing renal papillae for diffuse damage and contour rounding causing a billowy appearance leading to distal papillae blunting [12].

Concerning biological analyses, the metabolic and phosphorus-calcium workups were done in our patient with no significant abnormalities. It has been demonstrated that MSK is usually associated with increased levels of urinary calcium along with normal urinary potassium and bicarbonate levels in most patients. Urine $\mathrm{pH}$, ammonia and titratable acid are increased in MSK patients [12].

Mc Phail et al. [4] conducted a study that aimed to evaluate clinical and metabolic features in 56 patients with MSK. They found that stones were composed of calcium oxalate monohydrate, calcium oxalate dihydrate, calcium phosphate apatite, and uric acid. Metabolic profile was obtained for 26 of 39 (67\%) stoneforming patients demonstrating abnormalities in 22/26 (84.6\%). These included hypercalciuria, 58\% (15/26); low urine volume, 35\% (9/26); hyperuricosuria, $27 \%$ (7/26); hypocitraturia, 19\% (5/26); elevated urine sodium, 15\% (4/26); and hyperoxaluria, $12 \%(3 / 26)$.

Faced with calcification and total obstruction of the left kidney, nephrectomy was performed in our patient. It has been suggested that if MSK is not diagnosed early leading to long term irreversible complications, nephrectomy may be required [11]. Cheungpasitporn et al. [13] while studying the outcomes of living kidney donors with medullary sponge kidney found that donors with MSK had lower kidney stone-related events after nephrectomy compared with nondonors with MSK. Meanwhile, the right kidney of our patient was already a site for stone formation. This could be explained by the fact that MSK is a congenital disease that may affect both kidneys [14].

We found various types and sizes of stones in the removed kidney of our patient. However, these stones have not been analyzed due to an insufficient technical platform. Meanwhile, we identified staghorn calculi. This may suggest infectious episodes that may have gone unnoticed. Gambaro et al. argued that infectious episodes may complicate the renal stones, leading occasionally to secondary struvite stones and/or pyelonephritis, which are probably the most common causes of end stage renal disease in MSK patients [15].

The main goal of treatment of MSK is the prevention of recurrent urolithiasis and inhibition of progression of nephrocalcinosis. In all patients, oral potassium citrate supplementation and high fluid intake are indicated [16] [17].

According to Fabris et al. [3], after studying a cohort of 61 MSK patients, made the following recommendations: oral potassium citrate $(2-4 \mathrm{~g} / \mathrm{day}$, i.e. 10 - $20 \mathrm{mmol} /$ day divided into two or three doses) in patients who have MSK and at 
least one urine abnormality (hypercalciuria, hypocitraturia, hyperuricosuria and/ or hyperoxaluria).

Start with $2 \mathrm{~g}$ /day and gradually step up the dose in patients who fail to achieve the target citraturia level of $450 \mathrm{mg} / 24 \mathrm{~h}$, adding $1 \mathrm{~g}$ at a time until this citraturia level is reached, provided the urinary $\mathrm{pH}$ in a $24-\mathrm{H}$ collection is $<7.5$ (to prevent the risk of exacerbating any calcium phosphate lithogenesis).

Patients are followed up once a month until the treatment's dosage has been finely adjusted, then 6-monthly [3].

Other prophylactic measures, such as increasing water intake, reducing dietary sodium and proteins, increasing vegetable and fruit intake, are also generally recommended [3].

Aside from recurrent episodes of nephrolithiasis, MSK can be considered a benign condition. Typical complications of nephrolithiasis are observed in patients including UTIs, pyelonephritis, and end stage renal disease, especially if urinary tract obstruction is not addressed timely [5] [13].

In case of urinary tract obstruction, the use of minimally-invasive procedures may be useful. As a last resort, surgical intervention consisting of open stone extraction or nephrectomy may be an option for patients who experience both recurrent stone formation and severe symptoms. Though stone removal is necessary to relieve symptoms, the actual prognosis of the disease does not change [3] [5].

Compliance with all these measures and collegial follow-up in a nephrourology unit would delay or avoid progression to renal failure.

\section{Conclusion}

Nephrocalcinosis and nephrolithiasis are complications of MSK. Its asymptomatic character can often lead to diagnostic delay. Any telltale signs such as hematuria, urinary tract infections, or renal colic should lead to proper imaging to exclude Cacchi Ricci disease. Dietary and hygiene measures may be sufficient to avoid nephrectomy and delay chronic kidney disease. For this purpose, it is recommended that MSK patients with at least one stone risk factor (hypercalciuria, hypocitraturia, hyperuricosuria, hyperoxaluria) are considered to be at a higher risk for renal stone complications and should prophylactically be treated with potassium citrate [18].

\section{Informed Consent}

Thorough consent was formally obtained from the patient to report on this case.

\section{Authors' Contributions}

All authors contributed to the realization of this manuscript

\section{Conflicts of Interest}

The authors declare no competing financial or personal interests. 


\section{References}

[1] Geavlete, P., Nita, G, Alexandrescu, E. and Geavlete, B. (2013) The Impact of Modern Endourological Techniques in the Treatment of a Century Old DiseaseMedullary Sponge Kidney with Associated Nephrolithiasis. Journal of Medicine and Life, 6, 482-485.

[2] Gambaro, G., Danzab, F.M. and Fabrisc, A. (2013) Medullary Sponge Kidney. Current Opinion in Nephrology and Hypertension, 22, 421-426. https://doi.org/10.1097/MNH.0b013e3283622b86

[3] Fabris, A., Lupo, A. and Gambaro, G. (2012) Medullary Sponge Kidney: State of the Art. Nephrology Dialysis Transplanation, 28, 1111-1119. https://doi.org/10.1093/ndt/gfs505

[4] McPhail, E.F., Gettman, M.T., Patterson, D.E., Rangel, L.J. and Krambeck, A.E. (2012) Nephrolithiasis in Medullary Sponge Kidney: Evaluation of Clinical and Metabolic Features. Urology, 79, 277-281. https://doi.org/10.1016/j.urology.2011.07.1414

[5] Talha, H.I., Haris, P. and Hassan, P. (2019) Medullary Sponge Kidney: Current Perspectives. The International Journal of Nephrology and Renovascular Disease, 12, 213-218. https://doi.org/10.2147/IJNRD.S169336

[6] Kass-Iliyya, A. and Young, J.G. (2016) Medullary Sponge Kidney: A Treatment Conundrum. Journal of Clinical Urology, 9, 193-200. https://doi.org/10.1177/2051415816632075

[7] Kusz, M., Bienias, B., Wieczorkiewicz-Płaza, A., Brodzisz, A., Wieczorek, P. and Sikora, P. (2019) Nephrocalcinosis in Adolescent Girl with Medullary Sponge Kidney and Mild Hemihypertrophy. Medicine, 98, e14529.

https://doi.org/10.1097/MD.0000000000014529

[8] Gaunay, G.S., Berkenblit, R.G., Tabib, C.H., Blitstein, J.R., Patel, M. and Hoenig, D.M. (2017) Efficacy of Multi-Detector Computed Tomography for the Diagnosis of Medullary Sponge Kidney. Current Urology Reports, 11,139-143. https://doi.org/10.1159/000447208

[9] Giambelluca, D., Caruana, G., Giambelluca, E., et al. (2018) The "Bouquet of Flowers” Appearance in Medullary Sponge Kidney. Abdominal Radiology, 43, 2206-2207. https://doi.org/10.1007/s00261-017-1420-0

[10] Xiang, H., Han, J., Ridley, W.E. and Ridley, L.J. (2018) Medullary Sponge Kidney. Journal of Medical Imaging Radiation Oncology, 62, 93-94. https://doi.org/10.1111/1754-9485.40_12784

[11] Cheungpasitporn, W. and Erickson, S. (2015) Medullary Sponge Kidneys and the Use of Dual-Energy Computed Tomography. Urology Annals, 7, 129 p. https://doi.org/10.4103/0974-7796.148666

[12] Evan, A.P., Worcester, E.M., Williams, J.C., et al. (2015) Biopsy Proven Medullary Sponge Kidney: Clinical Findings, Histopathology, and Role of Osteogenesis in Stone and Plaque Formation: Pathogenesis of MSK Stones. The Anatomical Record, 298, 865-877. https://doi.org/10.1002/ar.23105

[13] Cheungpasitporn, W., Thongprayoon, C., Brabec, B.A., Kittanamongkolchai, W. and Erickson, S.B. (2016) Outcomes of Living Kidney Donors with Medullary Sponge Kidney. Clinical Kidney Journal, 9, 866-870. https://doi.org/10.1093/ckj/sfv107

[14] Fick, G.M. and Gabow, P.A. (1994) Hereditary and Acquired Cystic Disease of the Kidney. Kidney International, 46, 951-964. https://doi.org/10.1038/ki.1994.354

[15] Gambaro, G., Favaro, S. and D’Angelo, A. (2001) Risk for Renal Failure in Nephro- 
lithiasis. American Journal Kidney Diseases, 37, 233-243.

https://doi.org/10.1053/ajkd.2001.21285

[16] Mayall, G.F. (1970) The Incidence of Medullary Sponge Kidney. Clinical Radiology, 21,171-174. https://doi.org/10.1016/S0009-9260(70)80111-8

[17] Fabris, A., Bernich, P., Abaterusso, C., et al. (2009) Bone Disease in Medullary Sponge Kidney and Effect of Potassium Citrate Treatment. Clinical Journal of the American Society of Nephrology, 4, 1974-1979. https://doi.org/10.2215/CJN.02360409

[18] Fabris, A., Lupo, A., Bernich, P., et al. (2010) Long-Term Treatment with Potassium Citrate and Renal Stones in Medullary Sponge Kidney. Clinical Journal of the American Society of Nephrology, 5, 1663-1668.

https://doi.org/10.2215/CJN.00220110 